\title{
Hebelpunkte lokaler Ökonomien. Der Betrieberückgang im lokalen Lebensmittelhandwerk aus systemischer Perspektive
}

\author{
David Sipple (10 - Heiner Schanz (1) \\ Eingegangen: 9. Juni 2020 - Angenommen: 22. Dezember 2020 - Online veröffentlicht: 9. Februar 2021
}

\begin{abstract}
Zusammenfassung
Vielfältige lokale Ökonomien sind ein zentraler Faktor für das Gelingen von Nachhaltigkeitstransformationen auf kommunaler Ebene. Doch gerade die heterogenen Strukturen inhabergeführter, ortsansässiger (Klein-)Betriebe scheinen in ihrer Existenz von voranschreitenden überregionalen Marktkonzentrations- und Filialisierungsprozessen bedroht. Gleichzeitig haben kommunale Planung, Verwaltung und Politik bisher nicht alle Bereiche lokaler Ökonomien vollumfänglich im Blick. Die Suche nach Hebelpunkten für Governance-Interventionen zur Förderung und zum Erhalt lokaler Ökonomien erfordert mehr Aufmerksamkeit. Auch das Fallbeispiel des vorliegenden Beitrags, das inhabergeführte, ortsansässige Lebensmittelhandwerk, ist von existenziellen Schwierigkeiten bedroht. Über qualitative Experteninterviews mit Bäckereien und Metzgereien in zwei süddeutschen Mittelstädten wird den Ursachen des Betrieberückgangs und diesbezüglicher betriebsinterner Strategien nachgegangen. Dies erfolgt aus systemischer Perspektive, in Form der Modellierung von Kausalschleifendiagrammen und der Einordnung dieser in die Typologie sogenannter Systemarchetypen. Ziel des Beitrags ist die Identifikation von Hebelpunkten für Governance-Interventionen, über welche die Stadtentwicklung die Branchen
\end{abstract}

David Sipple, Professur für Environmental Governance, Albert-Ludwigs-Universität Freiburg, Tennenbacher Straße 4, 79106 Freiburg, Deutschland

david.sipple@envgov.uni-freiburg.de

Prof. Dr. Heiner Schanz, Professur für Environmental Governance, Albert-Ludwigs-Universität Freiburg, Tennenbacher Straße 4, 79106 Freiburg, Deutschland heiner.schanz@envgov.uni-freiburg.de

( 2021 Sipple; licensee oekom verlag. This Open Access article is published under the Creative Commons Attribution-ShareAlike 4.0 International Licence. einer kleinteiligeren lokalen Ökonomie gezielt unterstützen kann.

Schlüsselwörter: Lokale Ökonomie Nachhaltigkeitstransformation - Stadtentwicklung = Stadtplanung - kommunale Wirtschaftsförderung nachhaltige Ernährungssysteme

Leverage points of local economies. Business closures in local artisan food production from a system perspective

\begin{abstract}
The diversity of local economies represents a central factor for the success of sustainability transformations at municipal levels. But especially the heterogeneous structures of familyowned, locally based (small-scale) businesses seem to be threatened in their existence by market concentration and branching processes. At the same time, municipal planning departments as well as local administrations and politics have not yet taken account of all sectors of local economies and their differing needs. Searching levers for governance interventions to promote and maintain local economies therefore requires more attention. Family-owned, locally based (smallscale) food trade businesses, which represent the case study of this paper, are currently exposed to existential threats. In a first step, the causes of the business closures and the accompanying expectations and strategic considerations are analysed via qualitative expert interviews with bakeries and butchers in two medium-sized towns in Southern Germany. This is done from a systemic perspective, in the form of modelling causal loop diagrams and the classification of these into so-called system archetypes. The aim is, in a second step, to identify leverage points for governance interventions,
\end{abstract}


through which local authorities can support the branches of the (small-scale) local economy.

Keywords: Local economy - Sustainability transformation * Urban development - Urban planning - Municipal business development $\mathbf{-}$ Sustainable food systems

\section{Einleitung und Problemstellung}

Lokale Ökonomien sind nicht nur charakteristische, sondern auch wesentlich konstituierende Elemente von Städten und Gemeinden (Sperle 2012: 68-69). Als lokale Ökonomie wird dabei der Bereich der städtischen Wirtschaft bezeichnet, „der über vielfältige Vermittlungsformen in die ökonomischen und sozialen Strukturen und Interaktionsbeziehungen von Stadtteilen oder Stadtquartieren ,eingebet-

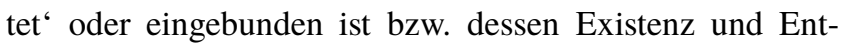
wicklung von diesen Einbettungsformen abhängt" (Läpple/ Walter 2003: 24). Die lokale Einbindung manifestiert sich im Unternehmenstyp, der vor allem inhabergeführte, kleine Einzelhandels- und Handwerksunternehmen einschließt, ihrem unmittelbaren regionalen Raumbezug und ihrer primär auf die ortsansässige Kundschaft gerichtete Versorgungsfunktion, ihre lokale Bindung von Wertschöpfung sowie ihrer prägenden Funktion für das Stadtbild (Helmle 2005; Henn/Behling 2020: 12). Die lokale Ökonomie umfasst vier grundlegende Funktionen des städtischen Lebens: (1) Beschäftigungs- und Ausbildungsfunktion, (2) Versorgungsfunktion, (3) Kommunikations- und Integrationsfunktion, (4) Aufwertungsfunktion (Jakubowski/Koch 2009: 242).

Inhabergeführte, unabhängige Unternehmen haben positive Effekte auf die Funktionen lokaler Ökonomien (Franz 2020: 205) und gelten durch ihre unmittelbare Versorgungsfunktion für die Stadtbevölkerung als ein zentraler Versorgungsbereich (Henn/Behling 2020: 12). Dies gilt auch für das ortsansässige, inhabergeführte Lebensmittelhandwerk. Es umfasst zulassungsfreie Gewerke der Mühlen, Brauereien und Mälzereien sowie zulassungspflichtige Handwerksberufe in der Bäckerei, Konditorei und Metzgerei (Destatis 2020: 19). Im Laufe der letzten zehn Jahre nahm die Zahl der Lebensmittelhandwerksbetriebe in Deutschland um fast $25 \%$ ab. ${ }^{1}$ Besonders betroffen sind die Betriebe des Bäcker- und Metzgereihandwerks, welche in den vergangenen zehn Jahren um jeweils $30 \%$ zurückgingen. Im gleichen Zeitraum nahm der Umsatz pro Betrieb um jeweils rund $50 \% \mathrm{zu}$, was auf eine voranschreitende Marktkonzentrati-

\footnotetext{
1 https://www.zdh-statistik.de/application/index.php?mID=3\& CID=422 (09.12.2020).
}

on und zunehmende Filialisierung hinweist (Statista 2019a: 26; Statista 2019b: 10).

Es zeigt sich insgesamt ein wachsender Konkurrenzdruck unter anderem durch Tankstellenshops, filialisierte Großbetriebe sowie die Backautomaten und Fleischtheken des konzentrierten Lebensmitteleinzelhandels (Kögl/Tietze 2010: 65). Diese Großunternehmen machen sich automatisierte und zentralisierte Verarbeitungsstrukturen zunutze, welche betriebskostensenkende Skalenerträge erzeugen (Bizer/Haverkamp 2012: 7; Deutscher Bundestag 2019: 4). Gleichzeitig fehlt inhabergeführten Kleinbetrieben häufig das Investitionskapital für notwendige Vergrößerungen, Spezialisierungen und Modernisierungen (Beile/DrescherBonny/Maack 2009: 13-14), weshalb ihre Marktanteile schwinden (Rohn 2012: 128).

Durch den voranschreitenden Betrieberückgang im ortsansässigen, inhabergeführten Lebensmittelhandwerk droht ein sukzessiver Verlust der individuellen Nahversorgungsfunktion von Ortskernen und Innenstädten, was auch aus Sicht der Stadtentwicklung problematisch ist (Runst/ Haverkamp 2018). Die Folge ist ein Attraktivitätsverlust der Zentren, welcher sich in einer Konzentration und Uniformität des Angebots, zunehmender Filialisierung und Textilisierung und wachsenden Leerstandsquoten äußert, was letztlich in eine sinkende Passantenfrequenz mündet (Mossig/Dorenkamp 2010: 3). Es droht eine soziale und wirtschaftliche ,(Innen-)Entwertung“ der Ortskerne, welche einen Verlust der Funktion sowie des individuellen Charakters der Innenstädte zugunsten randstädtischer Subzentren mit sich bringt (Ulm 2007: 156). Diese in der räumlichen Planung mit „Trading-Down“ beschriebene Entwicklung erfasst besonders Mittel- und Kleinstädte (Dannenberg/Willkomm/Zehner 2017: 4). Während solche Prozesse relativ breit verstanden werden und beschrieben sind, basieren viele der in Forschung und Praxis beschriebenen Steuerungsmaßnahmen, mit welchen diesen selbstverstärkenden Entwicklungen effektiv entgegengewirkt werden soll, auf deskriptiven Einzelfallstudien (z. B. Weck 2012; Brandt/Gärtner 2019). Systematische Analysen von Ansatzpunkten und Wirkungszusammenhängen aus steuerungstheoretischer Perspektive fehlen bisher weitestgehend (Spars/Busch 2020: 34-35).

Gleichzeitig kommt insbesondere Städten und Gemeinden eine Schlüsselrolle bei der Implementierung der im Rahmen der „Agenda 2030 für nachhaltige Entwicklung“ von den Vereinten Nationen (2015) verabschiedeten Ziele für nachhaltige Entwicklung zu, da sich auf ihrer Ebene ökonomische, ökologische und soziale Prozesse konkretisieren und (re-)produzieren (Dütschke/Köhler/Laws et al. 2019: 80-82). Mit den sogenannten Sustainable Develop- 
ment Goals ${ }^{2}$ soll eine globale ,Nachhaltigkeitstransformation“ angestoßen werden (Leal Filho 2019: 2). Die Zukunftsfähigkeit von lokalen Ökonomien ist deshalb unmittelbar mit politischen Nachhaltigkeitszielen und den dafür notwendigen gesellschaftlichen Transformationen verbunden (Henn/Behling 2020: 20). Innerhalb von lokalen Ökonomien scheinen insbesondere inhabergeführte Betriebe, wie die des Lebensmittelhandwerks, eine wichtige Voraussetzung für die Umsetzung einer nachhaltigen Stadt- und Raumentwicklung zu sein (Jakubowski/Koch 2009: 241). Dies zeigen Untersuchungen der lokalen Tourismus- (Luthe/Wyss/Schuckert 2012: 849) und Ernährungswirtschaft (Sloane/O'Reilly 2013: 634). Das inhabergeführte Lebensmittelhandwerk bietet dabei aufgrund seiner zentralen wie auch vermittelnden Rollen einen wichtigen Hebel für Systemtransitionen im Kontext gesellschaftlicher Nachhaltigkeitstransformationen (Sipple/Schanz 2019).

Trotz einer offensichtlichen Bedeutung von systemischen Wechselwirkungen und Rückkopplungen zur Erreichung von normativen Entwicklungszielen dienen bisher nur einfache Interpretationen der Umsatz-, Nachfrage- und Absatzentwicklungen als Grundlage für Politikempfehlungen zur Unterstützung des ortsansässigen, inhabergeführten Lebensmittelhandwerks (vgl. Haverkamp 2011). Die Nichtberücksichtigung von Rückkopplungen bei der Ableitung von Politikempfehlungen für Governance-Interventionen beschränkt sich jedoch nicht auf einzelne Teilbereiche oder Branchen, sondern betrifft die Frage nach Hebelpunkten in lokalen Ökonomien ganz generell. In der hier vorgestellten Studie $^{3}$ wurde versucht, sich dieser Problemstellung explorativ zu nähern, indem am Beispiel des Rückgangs der Betriebe im ortsansässigen, inhabergeführten Lebensmittelhandwerk zweier süddeutscher Mittelstädte Ursachen identifiziert und Hebelpunkte zum Erhalt abgeleitet wurden. Entsprechend sind die Themen des Beitrags:

- Identifikation der als zentral wahrgenommenen Ursachen und Wirkungen des Betrieberückgangs

- Analyse diesbezüglicher betriebsinterner Interventionen und ihrer Auswirkungen

- Identifikation wirksamer Governance-Interventionen für die Stadtentwicklung - auf Basis der Analyse - über die Ableitung sogenannter Systemarchetypen und zugehöriger Lösungsstrategien (Senge 2011: 113-115)

\footnotetext{
2 https://sdgs.un.org/goals (09.12.2020).

3 Die Studie ist Teil des transdisziplinären Verbundforschungsprojekts KERNiG, gefördert durch das Bundesministerium für Bildung und Forschung (BMBF) im Rahmenprogramm „FONA - Forschung für Nachhaltige Entwicklung“ (Förderkennzeichen: 01UR1614A).
}

Die empirische Erhebung basiert auf 16 qualitativen Experteninterviews mit Inhaberinnen und Inhabern ortsansässiger Lebensmittelhandwerksbetriebe und erfolgte eingebettet in das Verbundforschungsprojekt KERNiG zur Frage der Governance von kommunalen Ernährungssystemen am Beispiel der zwei süddeutschen Mittelstädte Waldkirch im Breisgau und Leutkirch im Allgäu (Schanz/Pregernig/Baldy et al. 2020).

Der Beitrag geht zunächst auf die systemische Perspektive von Interventionsdynamiken als theoretisch-konzeptionelle Grundlage ein (Kapitel 2). Es folgt die Abgrenzung der Untersuchungsräume und die Erläuterung der Methodik der Datenerhebung, -auswertung und -modellierung (Kapitel 3). Im Ergebnisteil (Kapitel 4) werden sogenannte Systemarchetypen aufgezeigt sowie Lösungsstrategien abgeleitet. Der Beitrag schließt mit einer Diskussion (Kapitel 5) und einem Resümee der zentralen Ergebnisse (Kapitel 6).

\section{Systemische Perspektive - Hebelpunkte für Interventionen}

Die Analyse von Systemdynamiken zielt darauf ab, zeitabhängige, dynamische Verhaltensmuster eines Systems zu identifizieren und über eine qualitative und/oder quantitative Modellierung zu simulieren, zu verstehen und letztlich zu optimieren (Coyle 1997: 10). Dabei sollen explizit die Variablen endogener, systemimmanenter Dynamiken offengelegt werden, welche über kausale Abhängigkeiten miteinander verknüpft sind (Richardson 2011: 224). Die Abgrenzung des zu analysierenden Systems ergibt sich aus der zugrundeliegenden Problem- bzw. Fragestellung, das heißt wie im Fall der vorliegenden Untersuchung aus der räumlich-administrativen Transitionsebene (van Raak 2015: 277278). Die Analyse zielt ferner darauf $a b$, die entsprechenden mentalen Modelle zu identifizieren, über welche sich Individuen die Funktionsweisen ihrer (Betriebs-)Realität erklären und sie damit strukturieren. Menschen und soziale Gruppen verfügen über ein besonderes Verständnis für die Wirkzusammenhänge, welche sie durch ihre Handlungen und soziale Interaktion (re-)produzieren (Mingers 2014: 6-7). Über eine systemische Modellierung dieser Vorstellungen können daher mögliche „Hebelpunkte“ (leverage points) identifiziert werden, um Systeme in eine gewünschte Richtung zu bewegen (Meadows 2019: 211).

Als Hebelpunkte werden die Stellschrauben in einem System verstanden, durch deren Veränderung ein Wandel bewirkt werden kann. Dabei gilt zu berücksichtigen, dass komplexe Systeme häufig kontraintuitiv sind: Bewusste Entscheidungen, ein System zu verändern, führen nicht zwangsläufig zum gewünschten Erfolg in Form von (ausschließlich) 
Tabelle 1 Interventionskategorien und Hebelpunkte der Systemintervention

\begin{tabular}{|c|c|c|c|}
\hline & & Beschreibung & Hebelpunkte \\
\hline \multirow[t]{4}{*}{$\begin{array}{l}\text { Interventions- } \\
\text { kategorien }\end{array}$} & Parameter & $\begin{array}{l}\text { Physische, quantifizierbare Hebel- } \\
\text { punkte }\end{array}$ & $\begin{array}{l}\text { 1. Zahlen: Konstanten und Parameter } \\
\text { 2. Puffer: Verhältnis zwischen Beständen und Flüssen } \\
\text { 3. Systemstrukturen mit Beständen und Flüssen }\end{array}$ \\
\hline & $\begin{array}{l}\text { Rück- } \\
\text { kopplungen }\end{array}$ & $\begin{array}{l}\text { Wechselwirkungen, die Rückkopp- } \\
\text { lungen beeinflussen und/oder den } \\
\text { (Miss-)Erfolg einer Intervention auf- } \\
\text { zeigen }\end{array}$ & $\begin{array}{l}\text { 4. Verzögerungen: Geschwindigkeit der Systemveränderung } \\
\text { 5. Ausgleichende Rückkopplung: balancierende Wirkung im } \\
\text { Verhältnis zu auslösenden Störfaktoren } \\
\text { 6. Selbstverstärkende Rückkopplung: Verstärkungsfaktor } \\
\text { von Kausalschleifen }\end{array}$ \\
\hline & Strukturen & $\begin{array}{l}\text { Soziale Strukturen und deren Aus- } \\
\text { wirkungen auf Informationsflüsse, } \\
\text { geltende Systemregeln und die Fä- } \\
\text { higkeit zur Selbstorganisation }\end{array}$ & $\begin{array}{l}\text { 7. Informationsflüsse: Zugang zu Informationen } \\
\text { 8. Regeln: Anreize, Strafen, Beschränkungen } \\
\text { 9. Selbstorganisation: Fähigkeit, Systemstrukturen zu } \\
\text { erweitern, zu verändern oder weiterzuentwickeln }\end{array}$ \\
\hline & Paradigmen & $\begin{array}{l}\text { Normen, Werte und Ziele, sprich } \\
\text { Paradigmen, aus denen ein System } \\
\text { hervorgeht }\end{array}$ & $\begin{array}{l}\text { 10. Ziele: Zweck oder die Funktion eines Systems } \\
\text { 11. Paradigmen: Vorstellungswelt, aus der ein System her- } \\
\text { vorgeht } \\
\text { 12. Paradigmen überwinden }\end{array}$ \\
\hline
\end{tabular}

Quelle: Eigene Darstellung nach Meadows (1999), Abson/Fischer/Leventon et al. (2017), Luederitz/Abson/Audet et al. (2017) und Meadows (2019)

positiven Folgen. Vielmehr erzeugen sie häufig (auch) negative Wirkungen. In Tabelle 1 sind die von Meadows (1999) entwickelten Hebelpunkte zur Systemintervention abgebildet. Da die vorliegende Studie an der Interventionskategorie der Rückkopplungen anknüpft, wurde diese kursiviert hervorgehoben.

Die nach ihrer Effektivität aufsteigend gelisteten Hebelpunkte beginnen mit der Interventionskategorie der Parameter. Als Hebelpunkte gelten sie als kurzfristig wirksam und werden deshalb besonders häufig durch die Politik angesprochen (Meadows 2019: 214-216). Bei Rückkopplungen handelt es sich um Dynamiken zwischen internen Systemvariablen, welche in ihrer Wirkung verzögert, selbstverstärkend und/oder balancierend sind (Meadows 2019: 219226). Strukturen beinhalten den Zugang zu Informationsflüssen sowie den Strukturen und Regeln eines Systems und deren Veränderbarkeit, weshalb sie grundlegend für ein Verständnis des Handelns systemrelevanter Akteure sind (Meadows 2019: 226-232). Als am wirksamsten gelten Paradigmen. Hierbei handelt es sich um grundsätzliche Annahmen sowie die Normen, Werte und Ziele eines Systems. Diesbezügliche Interventionen stellen deshalb die größte Herausforderung dar (Meadows 2019: 232-238).

Bisher wird die Ebene der Rückkopplungen zur Ableitung potenzieller Hebelpunkte für Governance-Interventionen kaum thematisiert, obwohl etwa die Bedeutung von Rebound-Effekten in ihren Wirkungen bekannt ist (Santarius 2014; Freeman/Yearworth/Preist 2016). Der Mehrwert der Interventionskategorie der Rückkopplungen für die kommunale Governance besteht darin, Hebelpunkte zur Systemintervention abzuleiten, in ihrer Wirksamkeit zu testen und ihre Funktionsweise verständlich zu kommuni- zieren (Hirschnitz-Garbers/Distelkamp/Koca et al. 2018). Vor allem kann aufgezeigt werden, dass von den Ausführenden als wirksam eingestufte Interventionen gegebenenfalls langfristig vom Ziel abweichende, unerwünschte oder gar gegensätzliche Rückkopplungen auslösen können. Auf die Identifikation solcher unerwarteter bzw. unerwünschter Effekte von Systeminterventionen und möglicher Lösungsstrategien zielt die vorliegende Analyse ab.

\section{Methodik}

\subsection{Untersuchungsräume}

Die Forschung zu Fragen der Governance kommunaler Ernährungssysteme in Deutschland erstreckt sich bislang hauptsächlich auf Großstädte wie Berlin, München, Nürnberg oder Freiburg (vgl. Fesenfeld 2016; Doernberg/Horn/ Zasada et al. 2019). Dabei sind rund 60\% der deutschen Bevölkerung in Städten und Gemeinden mit weniger als 50.000 Einwohner/-innen gemeldet. Unter den klassifizierten Stadt- und Gemeindetypen des Bundesinstituts für Bau-, Stadt- und Raumforschung (BBSR) bilden die ,kleineren Mittelstädte mit Zentrum (20.000 bis 49.999 Einwohner)“ mit einem Anteil von 19,3\% an der Gesamtbevölkerung die stärkste Kategorie. ${ }^{4}$ Die Größe der beiden Untersuchungsräume Leutkirch im Allgäu und Waldkirch im Breisgau

\footnotetext{
4 https://www.bbsr.bund.de/BBSR/DE/forschung/raumbeobachtung/downloads/downloadsReferenz2.html?nn=2544954 (14.12.2020).
} 
bilden mit je rund 23.000 Einwohner/-innen damit die Lebenswirklichkeit eines Großteils der deutschen Bevölkerung ab. Leutkirch verfügte als Flächengemeinde $\left(175 \mathrm{~km}^{2}\right)$ zum Zeitpunkt der Untersuchung über 266 landwirtschaftliche Betriebe, wohingegen Waldkirch $\left(48 \mathrm{~km}^{2}\right)$ aufgrund seiner Topographie, geprägt durch große Waldflächen und eine Tallage, 68 landwirtschaftliche Betriebe beheimatete (Statistisches Landesamt Baden-Württemberg 2017). Wie eine Analyse der Nahrungsmittelversorgung beider Kommunen zeigt, ist diese sehr ähnlich strukturiert. So gibt es in beiden Städten über acht Betriebe des filialisierten Lebensmitteleinzelhandels und sechs Bäckereifilialen (über-)regionaler Großbetriebe (Sipple/Schanz 2019: 6-10).

\subsection{Datenerhebung und Datenauswertung}

Das Sampling der vorliegenden Untersuchung besteht aus 16 qualitativen Experteninterviews. In Leutkirch im Allgäu gab es zum Zeitpunkt der Erhebung 2017 insgesamt elf Betriebe des ortsansässigen, inhabergeführten Lebensmittelhandwerks (sieben Bäckereien und vier Metzgereien), in Waldkirch im Breisgau sechs Betriebe (vier Bäckereien und zwei Metzgereien). Mit Ausnahme einer Bäckerei in Waldkirch konnten alle Betriebe interviewt werden.

Alle Interviewten fungieren als Inhaber/-innen und gleichzeitig Betriebsleiter/-innen, weshalb sie über spe- zifisches Fachwissen verfügen und somit eine Expertise für ihr Arbeitsfeld abgeben können (vgl. Meier Kruker/Rauh 2005: 65; Gläser/Laudel 2010: 12; Flick 2012: 214). Die Befragung konzentrierte sich auf alle Bäckerei- und Metzgereibetriebe, deren offizieller Unternehmenssitz zum Zeitpunkt der Erhebung auf der Gemarkung einer der beiden Kommunen lag. Das heißt nicht, dass die Gemeinden isoliert betrachtet werden, jedoch beziehen sich die Aussagen zur Wirkung von betriebsinternen Strategien, die Ableitung der Archetypen sowie potenzieller Hebelpunkte für Governance ausschließlich auf die kommunale Ebene. Räumlich und politisch übergeordnete Steuerungsbemühungen, wie etwa ordnungspolitische Maßnahmen, bleiben unberücksichtigt (vgl. Forrester 1969; Nabavi/Daniell/Najafi 2017).

Die qualitative Befragung folgte einem teilstandardisierten Fragebogen. Die Interviews wurden als Audiodateien aufgezeichnet und mit der Software MAXQDA ${ }^{\circledR}$ transkribiert sowie einer qualitativen Textanalyse nach Mayring (2010) unterzogen, was auch die Kodierung der Daten (offen, axial, selektiv) beinhaltete (vgl. Flick 2012: 388-396; Rädiker/Kuckartz 2019: 70-72). Als Kodierungsschema wurden die Wahrnehmungen und fiktionalen Erwartungen der Akteure nach Beckert $(2010 ; 2013)$ verwendet und zu zentral wahrgenommenen Systemvariablen verdichtet, die in einem weiteren Schritt als Grundlage für die Modellierung dienten. Aufgrund des explorativen Charakters der

\begin{tabular}{|c|c|}
\hline $\begin{array}{l}\text { Pfeile und Vorzeichen } \\
\text { - Über Pfeile wird die Kausalität zwischen zwei Variablen angezeigt } \\
\text { - Eine Veränderung in Variablen am Ursprung des Pfeils bewirkt eine } \\
\text { Änderung der Variablen am Ziel des Pfeils } \\
\text { - Ein Doppelstrich im Verbindungspfeil zeigt eine zeitlich verzögerte } \\
\text { Wirkung zwischen zwei Variablen an } \\
\text { - Ein „," neben der Pfeilspitze bedeutet, dass die Variable am Ursprung } \\
\text { des Pfeils die Variable am Ziel des Pfeils in die gleiche Richtung ändert } \\
\text { - Eine Zunahme in Variable } 1 \text { bedeutet eine Zunahme in Variable } 2(+/+) \\
\text { - Eine Abnahme in Variable } 1 \text { bedeutet eine Abnahme in Variable } 2(-/-) \\
\text { Variable } 1 \\
\text { - Ein ,"“neben der Pfeilspitze bedeutet, dass die Variable am Ursprung } \\
\text { des Pfeils die Variable am Ziel des Pfeils in entgegengesetzte Richtung } \\
\text { ändert } \\
\text { - Eine Zunahme in Variable } 1 \text { bedeutet eine Abnahme in Variable } 2(+/-) \\
\text { - Eine Abnahme in Variable } 1 \text { bedeutet eine Zunahme in Variable } 2(-/+) \\
\text { Variable } 1\end{array}$ & $\begin{array}{l}\text { Variablen und Schleifen } \\
\text { - Der Buchstabe } \mathbf{R}(R=\text { reinforcing) beschreibt eine selbstverstärkende } \\
\text { Dynamik, eine solche entsteht bei gerader Anzahl von „,“-Zeichen } \\
\text { innerhalb einer Schleife } \\
\text { - Es liegt ein Verhalten vor, das sich vom Gleichgewichtspunkt weg- } \\
\text { bewegt und ein systematisches Wachstum oder einen systematischen } \\
\text { Rückgang verursacht } \\
\text { - Der Buchstabe } \mathbf{B} \text { (B=balancing) beschreibt eine balancierende } \\
\text { Dynamik, eine solche entsteht bei ungerader Anzahl von ","-Zeichen } \\
\text { innerhalb einer Schleife } \\
\text { - Es liegt ein Verhalten vor, das sich in Richtung Gleichgewicht oder einer } \\
\text { Schwankung um das Gleichgewicht bewegt } \\
\text { Variable } 1 \\
\text { (B) Variable } 2 \\
\text { - Kausalschleifen bestehen aus abhängigen Variablen (gekrümmter Pfeil) } \\
\text { - Kausalschleifen können zudem durch unabhängige Variablen } \\
\text { beeinflusst sein: Variable } 1 \text { ist abhängig von Variable } 3 \text {, die vom System } \\
\text { unbeeinflusst ist (gerader Pfeil) }\end{array}$ \\
\hline
\end{tabular}

Abbildung 1 Funktionsweisen von Kausalschleifendiagrammen Quelle: Eigene Darstellung nach Sverdrup/Haraldsson/Olafsdottir et al. (2018: 38) und Moll (2019: 118-119) 
Systemperspektive wurde ein abduktives Vorgehen gewählt. Dabei wird vom erfassten und verdichteten Phänomen auf eine grundsätzliche Regelmäßigkeit geschlossen (Reichertz 2013: 72). Da die Analyse der Interviewdaten nur geringe inhaltliche Abweichungen zwischen beiden Kommunen sowie zwischen den Betriebsformen der Bäckereien und Metzgereien zeigte, wurden sowohl die beiden Städte als auch die Betriebsformen zusammengezogen. Es scheint sich hier um strukturelle Phänomene zu handeln, welche nur wenig regional- und gewerkespezifische Besonderheiten aufweisen.

\subsection{Datenanalyse mit Kausalschleifendiagrammen}

Zur Erfassung und Analyse von Strukturen und Rückkopplungen von Systemen hat sich die Modellierung mithilfe von Kausalschleifendiagrammen (CLD; Causal Loop Diagrams) bewährt (Holtz/Alkemade/de Haan et al. 2015: 54). Kausalschleifendiagramme sind als Sätze zu verstehen, die über die Verknüpfung von Schlüsselvariablen gebildet werden und durch die kausalen Zusammenhänge zwischen diesen einen Sinn ergeben (Kim 1992: 1). Mit dem Aneinanderreihen mehrerer kausaler Beziehungen, sogenannter Loops, entsteht eine zusammenhängende Erzählung über eine bestimmte Problemstellung (Sverdrup/Haraldsson/Olafsdottir et al. 2018: 36). Die Erstellung der Kausalschleifendiagramme erfolgte mit der Software Stella Architect ${ }^{\circledR}$. Abbildung 1 zeigt detailliert die Funktionsweise von Kausalschleifendiagrammen und die Ableitung von Rückkopplungen.

In einem weiteren Schritt wurden die erstellten Kausalschleifendiagramme anhand der Typologie in sogenannte Systemarchetypen eingeordnet und analysiert. Es handelt sich hierbei um eine Methode zur Charakterisierung generischer Ursache-Wirkungs-Strukturen hinter
Strategieentscheidungen in einem System (Senge 2011). Die Systemarchetypen basieren auf der Beschreibung des Zusammenwirkens von selbstverstärkenden und balancierenden Rückkopplungen, ausgehend von beabsichtigten Aktionen und (un-)beabsichtigten Reaktionen (Wolstenholme 2003: 8). Dabei gilt die Systemarchetypanalyse als empirisch überprüfbare Methodik zur kontextsensitiven Untersuchung mentaler Repräsentationen von systemrelevanten Entscheidungen (Eisenack/Villamayor-Tomas/Epstein et al. 2019; Oberlack/Sietz/Bürgi Bonanomi et al. 2019). Archetypen vereinfachen die Einordnung und das Verständnis komplexer Systemverhaltensweisen und ermöglichen eine nachvollziehbare Identifikation von Interventionsstrategien und Hebelpunkten (Setianto/Cameron/Gaughan 2014: 644). Senge (2011) beschreibt zehn maßgebliche Systemarchetypen: (1) die zeitverzögerte Balance, (2) die Eskalation, (3) Erfolg den Erfolgreichen, (4) Grenzen des Wachstums, (5) Problemverschiebung, (6) scheiternde Korrekturen, (7) abrutschende Ziele, (8) ungewollte Gegnerschaft, (9) Tragödie der Allmende, (10) Wachstum und Unterinvestition.

\section{Ergebnisse}

\subsection{Hauptursachen für den Betrieberückgang}

Die Auswertung der Interviewdaten führt zur Identifikation von drei unterschiedlichen Hauptursachen für den Betrieberückgang: (1) der herrschende Fachkräfte- und Nachfolgemangel, (2) ein Nachfragerückgang aufgrund der Marktkonzentration und daraus resultierendem Preisdruck durch Lebensmitteleinzelhandel und Filialbetriebe und (3) die durch massiven Betrieberückgang verzögert steigende Produktnachfrage bei Teilen der verbliebenen lokalen

Tabelle 2 Die zentral wahrgenommenen Systemvariablen aus der Sicht des ortsansässigen, inhabergeführten Lebensmittelhandwerks in den Mittelstädten Waldkirch und Leutkirch

\begin{tabular}{lll}
\hline Kodierfamilie $^{\mathbf{a}}$ & Codes $^{\mathbf{a}}$ & Abgeleitete Systemvariablen $^{\text {Fachkräfte- und }}$ \\
Nachfolgemangel & - Nachfolgemangel (94) & Arbeitsintensität; Attraktivität des Berufsfeldes; Auszubilden- \\
(238) & Übertragungseffekte (17) & de; familieninterne Nachfolge; externe Nachfolge; Fachkräfte; \\
& - Betriebsverkleinerungen: & Beratungsangebot; Öffnungszeiten lokaler Betriebe; Attrak- \\
& - Personalmangel (107) & tivität lokaler Betriebe; Konkurrenzvorteile lokaler Betriebe; \\
Produktnachfrage & - Personalkapazität (20) & Marketingmaßnahmen \\
(208) & - Konkurrenz Lebensmitteleinzelhandel/ & Betriebezahl pro Kommune; Produktnachfrage bei lokalen Be- \\
& Filialisten (46) & trieben; Produktpreis im Lebensmitteleinzelhandel und Filial- \\
& - Betrieberückgang (27) & bereich; wirtschaftlicher Erfolg anderer Branchen; Spezialisie- \\
& - Spezialisierung: & rungsbestrebungen: (a) Anteil regionaler Produkte, (b) Anteil \\
& - Beratung und Qualität (59) & biozertifizierter Produkte, (c) Qualität der Fachberatung; Sor- \\
& - Zertifizierung (34) & timentsbreite und -tiefe; produzierte Menge; Qualitätsvorteil; \\
& - Regionalisierung (42) & wahrgenommener Qualitätsvorteil; Kosten der Spezialisie- \\
& & rung; Lieferantensuche (bio/regional); bauliche/technische \\
& Investitionen; (Re-)Zertifizierung; Passantenfrequenz; Lokal- \\
& orientierte öffentliche Beschaffung
\end{tabular}

${ }^{a}$ die Zahl in den Klammern gibt die jeweilige Anzahl an kodierten Textstellen an 
Betriebe, welche dadurch an ihre Kapazitätsgrenzen kommen, was den Fachkräfte- und Nachfolgemangel verstärkt. Weitere Gründe, die einen Betrieberückgang beschleunigen können, wie beispielsweise steigende Mieten und verhältnismäßig niedrige Gehälter, sind damit nicht ausgeschlossen, wurden aber nicht als Hauptursachen genannt.

Aus den Schilderungen der Betriebsleiter/-innen lassen sich zwei teils gegenläufige, aber parallel bestehende Handlungsstrategien als Reaktion auf diese Ursachen ableiten: (1) Betriebsverkleinerungen als Reaktion auf den Fachkräfte- und Nachfolgemangel, (2) Spezialisierung auf potenziell ertragreichere Bereiche. Daraus leiten sich die in Tabelle 2 dargestellten Systemvariablen ab. Deren Ursache-Wirkungs-Beziehungen in Form von Kausalschleifendiagrammen sind in Abbildung 2 unter 4.2 dargestellt. Die identifizierten Systemvariablen sind im Folgenden in Kursivschrift hervorgehoben.

\subsection{Situation und Handlungsstrategien der Betriebe}

\subsubsection{Betriebsverkleinerung aufgrund des Fachkräfte- und Nachfolgemangels}

Ausgangssituation: Der herrschende Mangel an Auszubildenden, Fachkräften sowie familieninterner und externer Nachfolge geht im Falle der untersuchten Betriebe auf eine stetig rückläufige Attraktivität des Berufsfeldes zurück. Dies entspringt einer hohen Arbeitsintensität, aber auch einer starken Konkurrenz auf lokalen Arbeitsmärkten durch den wirtschaftlichen Erfolg anderer lokaler Branchen, welche im Gegensatz zu verhältnismäßig niedrigen Verdienstund Entwicklungsmöglichkeiten im ortsansässigen, inhabergeführten Lebensmittelhandwerk attraktive Löhne, Arbeitsbedingungen und Karriereoptionen vor Ort bieten. Der wirtschaftliche Erfolg anderer lokaler Branchen stärkt jedoch gleichzeitig die Produktnachfrage, z. B. wenn der lokale Tourismus- oder Industriesektor floriert, führt so aber auch zu zunehmender Betriebsauslastung. Ein Betrieberückgang im Allgemeinen hat auf lange Sicht eine steigende Produktnachfrage bei Teilen der verbliebenen Betriebe zur Folge und stärkt diese zwar (über die balancierende Rückkopplung B1), führt jedoch bei einem gleichbleibenden Mangel an Fachkräften zu einer weiteren Zunahme der Arbeitsintensität mit den bereits beschriebenen Folgen (über die selbstverstärkenden Rückkopplungen R1-R5).

Reaktion: Auf den Mangel an Fachkräften reagieren die befragten Betriebsleiter/-innen mit einer Einschränkung der Öffnungszeiten, um einen Rückgang der Arbeitsintensität zu erreichen. Dies führt zunächst zur gewünschten Reaktion (über die balancierende Rückkopplung B2), was den Fachkräftemangel entschärft. Einschränkungen der Öffnungszei- ten führen verzögert zu einer sinkenden Attraktivität lokaler Betriebe und einer Schwächung der Konkurrenzvorteile, woraus eine abnehmende Produktnachfrage und so eine Verstärkung des Betrieberückgangs resultieren (über die selbstverstärkende Rückkopplung R6).

Archetyp: Die Reaktion auf den Mangel an Fachkräften in Form der Einschränkung der Öffnungszeiten (Betriebsverkleinerung) fällt unter den Systemarchetyp der „Fehlkorrekturen“. Die vollzogene Korrektur in Form eines Rückgangs der Arbeitsintensität (über die balancierende Rückkopplung B2) ist nur vorübergehend erfolgreich. Langfristig führt sie zu einer ,ungewollten Konsequenz“ der rückläufigen Produktnachfrage bei lokalen Betrieben (über die selbstverstärkende Rückkopplung R6) (Senge 2011: 463).

\subsubsection{Spezialisierung 5 als Reaktion auf eine sinkende Produktnachfrage}

Ausgangssituation: Bevor ein voranschreitender Betrieberückgang bei Teilen der verbliebenen Betriebe $\mathrm{zu}$ einer steigenden Produktnachfrage führt (über die balancierende Rückkopplung B1), nimmt diese jedoch insgesamt ab, was besonders auf abnehmende Produktpreise im Lebensmitteleinzelhandel und Filialbereich zurückzuführen ist und durch eine vermehrt rückläufige Passantenfrequenz (unter anderem im Zuge eines beginnenden Trading-Downs) verstärkt wird. Eine sinkende Produktnachfrage führt zu einem verstärkten Betrieberückgang.

Reaktion: Durch die abnehmende Produktnachfrage verstärken sich bei den befragten Betrieben die Spezialisierungsbestrebungen. Diese zeigen sich in einer Spezialisierung der Betriebe auf die Erhöhung des (a) Anteils regionaler Produkte und/oder (b) Anteils biozertifizierter Produkte und/oder (c) der Qualität der Fachberatung, was eine geringere Sortimentsbreite und-tiefe und produzierte Menge zur Folge hat. Dies wirkt sich negativ auf die Attraktivität lokaler Betriebe und deren Konkurrenzvorteile aus, woraus eine abnehmende Produktnachfrage resultiert (über die selbstverstärkenden Rückkopplungen R8 und R9). Spezialisierungsbestrebungen bringen zudem Kosten der Spezialisierung in Form von Transaktionskosten (durch Lieferantensuche, (Re-)Zertifizierungskosten, Marketingmaßnahmen) und möglicherweise bauliche und/oder technische Investitionen mit sich, was eine Schwächung der Konkurrenzvorteile birgt und die Produktnachfrage weiter schwächt (über die selbstverstärkende Rückkopplung R7). Anderer-

\footnotetext{
${ }^{5}$ Marktstrukturen können mit Ökosystemen verglichen werden (vgl. Storbacka/Nenonen 2011), daher bietet eine Spezialisierung geschützte Entwicklungsmöglichkeiten, sogenannte Marktnischen (vgl. Smith/Raven 2012).
} 


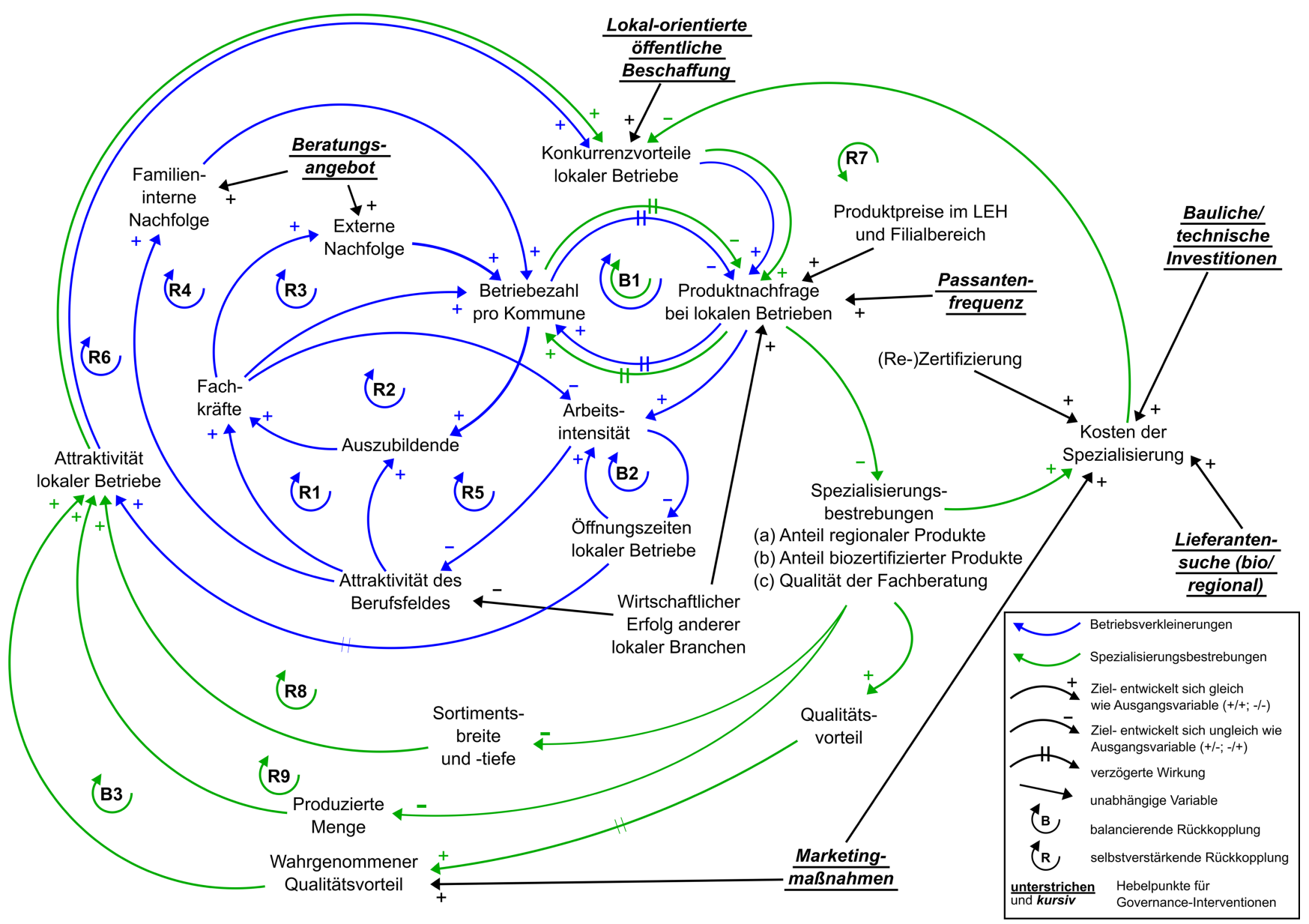

Abbildung 2 Gesamtmodell des Betrieberückgangs im ortsansässigen, inhabergeführten Lebensmittelhandwerk

seits führt eine Spezialisierung zu wachsenden Qualitätsvorteilen, die jedoch erst zeitlich verzögert von der Kundschaft wahrgenommen werden, dann aber eine Steigerung der Konkurrenzvorteile lokaler Betriebe und somit eine steigende Produktnachfrage zur Folge haben (über die balancierende Rückkopplung B3).

Archetyp: Zunächst führen Spezialisierungen zu selbstverstärkenden Rückkopplungen für die rückläufige Produktnachfrage (über die selbstverstärkenden Rückkopplungen R7-R9). Dies kann zur Folge haben, dass Betriebe diese Bestrebungen frühzeitig aufgeben. Sind die neugeschaffenen Verkaufsargumente jedoch einmal an die Kundschaft kommuniziert, entsteht ein Attraktivitätsgewinn für die Betriebe, welcher dem Rückgang der Betriebezahl pro Kommune entgegenwirkt (über die balancierende Rückkopplung B3). Die vorliegende Dynamik lässt sich daher mit dem Systemarchetyp „Gleichgewichtsprozess mit Verzögerung“ beschreiben, da die korrektive Aktion erst mit Verzögerung den gewünschten Erfolg zeigt (Senge 2011: 451).

\subsection{Wirkungsdynamiken und Hebelpunkte auf kommunaler Ebene}

Die verschiedenen, zumindest teilweise gegenläufigen Strategien bestehen parallel und können teilweise über gemeinsame Handlungsfelder und -punkte erreicht werden. Diese basieren auf den von Senge (2011) beschriebenen „Managementprinzipien“, mit welchen dem jeweiligen Archetyp begegnet werden kann (Setianto/Cameron/Gaughan 2014). Mithilfe dieser werden, auf der Empirie basierend, aus systemischer Perspektive Hebelpunkte für Kommunen abgeleitet, die zum Erhalt des ortsansässigen, inhabergeführten Lebensmittelhandwerks und so zu einer Stärkung lokaler Ökonomien beitragen (vgl. Abbildung 2). Sie sind in den Bereichen der Wirtschaftsförderung und Stadtplanung verortet.

Im Bereich der kommunalen Wirtschaftsförderung liegen die Hebelpunkte in den Tätigkeitsfeldern Beschäftigungsund Arbeitsmarktpolitik, Stadtmarketing sowie Cluster- und Netzwerkförderung: 
Tabelle 3 Strategie der Betriebe und daran angepasste Governance-Bereiche der Kommunen, Interventionsvariablen aus der Modellierung und Wirkungsziele

\begin{tabular}{llll}
\hline Strategie & Governance-Bereiche & Hebelpunkte & Wirkungsziel ( $\uparrow \downarrow$ ) \\
\hline Betriebsverkleinerung & Wirtschaftsförderung & & \\
& $\begin{array}{l}\text { Fachkräfte- und Nachfolgeförderung } \\
\text { Spezialisierung }\end{array}$ & $\begin{array}{l}\text { Beratungsangebote } \\
\text { Marketingmaßnahmen }\end{array}$ & $\begin{array}{c}\uparrow \uparrow \text { Attraktivität des Berufsfeldes } \\
\text { Stadtmarketing }\end{array}$ \\
& Wirtschaftsförderung & & \\
& Cluster- und Netzwerkförderung & Lieferantensuche & $\uparrow$ Wahrgenommene Qualitätsvorteile \\
& Stadtmarketing & Marketingmaßnahmen & $\downarrow$ Kosten der Spezialisierung \\
& Stadtplanung & & \\
& Bauvorschriften & bauliche/technische & $\downarrow$ Kosten der Spezialisierung \\
& Verkehrsplanung & Investitionen & $\uparrow$ Produktnachfrage \\
& Innenstadt- und Dorfentwicklung & Passantenfrequenz & \\
\hline
\end{tabular}

Ein Instrument dem Fachkräfte- und Nachfolgemangel entgegenzuwirken ist die Wirtschaftsförderung, indem Kampagnen des Stadtmarketings (Marketingmaßnahmen) auf eine Stärkung der Attraktivität des Berufsfeldes auf lokaler Ebene abzielen (schwächt R1-R5). Zudem können über ein Beratungsangebot bezüglich der Betriebsnachfolge betriebsspezifische Unterstützung durch die Kommune geleistet (schwächt R3 und R4) und Fördermöglichkeiten aufgezeigt werden. Wird die Verfügbarkeit von Fachkräften und die externe wie familieninterne Betriebsnachfolge auf diese Weise gestärkt, sind die Betriebe insgesamt weniger gefährdet, kurzfristige (Fehl-)Korrekturen wie im vorliegenden Archetyp vornehmen zu müssen (Senge 2011: 463; Systemarchetyp „Fehlkorrekturen“). Vergleichbare Unterstützungsangebote gibt es bereits in anderen Branchen (vgl. Franz 2020: 205). So scheint besonders die lokale Vernetzung sowie die Schaffung von Kontaktstellen für Personal- und Finanzierungsplanung hilfreich zu sein (Hilpert/ Völkening/Beck 2018: 113).

Die Spezialisierungsbestrebungen der Betriebe können ebenfalls über das Stadtmarketing (Marketingmaßnahmen) unterstützt werden, um die neu entstandenen Qualitätsvorteile schneller an die Kundschaft zu kommunizieren. So kann versucht werden, dem beschriebenen zunächst einsetzenden Nachfragerückgang entgegenzuwirken (stärkt B3). Über kommunale Markenbildung (sogenannte „Place making“-Maßnahmen) kann die Identität einer Kommune als Ort spezialisierter Nahrungsmittelproduktion und -verarbeitung gestärkt werden (Reiter 2018: 8). Auch die digitale Umsetzung lokaler Vermarktungs- und Vertriebsplattformen als zusätzliche Absatzkanäle (sogenanntes „Multi“und ,Cross-Channeling“) bietet hierbei Potenziale (Hilpert/ Völkening/Beck 2018: 113). Auf diese Weise können die Kosten der Spezialisierung der Betriebe verringert werden (schwächt R7). Gerade in inhabergeführten Kleinbetrieben fehlt es an zeitlichen Ressourcen und Fähigkeiten, solche innovativen Vermarktungskonzepte aufzubauen, weshalb hier die Kommunen gefordert sind (Proeger/Thonipara/ Bizer 2020: 17-18).

Zudem zeigt die Modellierung, dass Spezialisierungsbestrebungen über das Instrument der Cluster- und Netzwerkförderung unterstützt werden können. Über themenund branchenspezifische Netzwerktreffen (Chilla/Kühne/ Neufeld 2016: 139) werden lokale Netzwerke gestärkt und die Transaktionskosten beteiligter Betriebe gesenkt (Kulke 2017: 54), da so die Lieferantensuche vereinfacht und Wissens- und Erfahrungsaustausch ermöglicht wird. Dies verringert die Kosten der Spezialisierung (schwächt R7), weshalb tendenziell weniger Betriebe diese verfrüht aufgeben (Senge 2011: 463; Systemarchetyp „Gleichgewichtsprozess mit Verzögerung“). Über eine lokal-orientierte öffentliche Beschaffung (z. B. Verpflegung in kommunalen Bildungsund Pflegeeinrichtungen) können Spezialisierungsbestrebungen unterstützt und Konkurrenzvorteile lokaler Betriebe gestärkt werden (schwächt R7-R8), wie das ,Netzwerk deutscher Biostädte" ${ }^{\text {" }}$ zeigt (Fülles/Roehl/Strassner et al. 2017).

Im Bereich der Stadtplanung sind vor allem die Instrumente der Bauvorschriften sowie der Innenstadt- und Dorfentwicklung von Relevanz: Maßnahmen der Innenstadtentwicklung zielen auf eine Revitalisierung der Innenstädte und somit auf eine stärkere Passantenfrequenz. Dies kann über Maßnahmen wie einheitliche Öffnungszeiten, Leerstandsmanagement, Parkraummanagement, eine Beschilderung zu lokalen Betrieben in Verbindung mit einer Verbesserung der Fußgängersituation und Aufenthaltsqualität in der Innenstadt (z. B. durch Verkehrsberuhigung) erreicht werden (Hilpert/Völkening/Beck 2018: 114-115). So kann „Trading Down“-Effekten entgegengewirkt und eine positive Wirkung auf die Produktnachfrage erreicht werden.

Über eine Anpassung der ,klassischen“ Instrumente der Stadtplanung, wie der Bebauungsplan, aber auch über infor-

\footnotetext{
6 https://www.biostaedte.de/ (20.12.2020).
} 
melle Pläne und Konzepte (z. B. kommunale (Fach-)Konzepte zur Erhöhung der Nutzungsvielfalt) können sowohl die Passantenfrequenz als auch die Spezialisierungsbestrebungen unterstützt werden (zur Nedden/Bunzel/Pätzold et al. 2015: 103-107). Diesbezügliche Anpassungen können die Kosten der Spezialisierung verringern, indem sie anfallende bauliche/technische Investitionen ermöglichen (schwächt R7). Dies kann dazu beitragen, dass Spezialisierungen eher beibehalten werden (Senge 2011: 463; Systemarchetyp „Gleichgewichtsprozess mit Verzögerung“).

Zusammenfassend lassen sich zwei Governance-Bereiche mit insgesamt fünf Hebelpunkten zur Stärkung des ortsansässigen, inhabergeführten Lebensmittelhandwerks auf kommunaler Ebene ableiten (vgl. Tabelle 3).

Aus der Sicht der kommunalen (Innen)stadtentwicklung sind Governance-Interventionen zur Bekämpfung des Fachkräfte- und Nachfolgemangels sowie zur Unterstützung von Spezialisierungsbestrebungen und zum Erhalt des ortsansässigen, inhabergeführten Lebensmittelhandwerks im Allgemeinen ein wichtiger Baustein, um einem strukturellen Verfall ihrer Innenstädte und Ortskerne entgegenzuwirken (unter anderem „Trading Down“). So können Maßnahmen in allen analysierten Bereichen zu einer Absicherung bestehender und zur Schaffung neuer Ausbildungs- und Arbeitsplätze führen sowie zum Erhalt bzw. zur Verbesserung der Nahversorgungssituation beitragen und so die Heterogenität lokaler Ökonomien stärken. Dies kann zudem, speziell im Bereich der ökologischen/regionalen Spezialisierung, positive Effekte auf die Nachhaltigkeitstransformation des kommunalen Ernährungssystems haben, auch weil die inhabergeführten Betriebe weiterhin als wichtige Ansprechpartner mit Gestaltungspotenzial vor Ort existent sind.

\section{Diskussion}

Die Ergebnisse des vorliegenden Beitrags zeigen, dass es entgegen der häufigen Forderung nach ordnungspolitischen Maßnahmen und der notwendigen Änderungen im Konsumentenverhalten durchaus auch auf kommunaler Ebene effektive Möglichkeiten gibt, lokale Ökonomien gezielt zu stärken. Dabei wird die Notwendigkeit eines gezielten Einsatzes der Instrumente der endogenen Raumwirtschaftspolitik in Form der kommunalen Wirtschaftsförderung (direkte Instrumente) und der Maßnahmen der Stadtplanung (indirekte Instrumente) deutlich (Kulke 2017: 46-47).

Die kommunale Wirtschaftsförderung gilt ,als wesentlicher und integraler Bestandteil der Standortentwicklungspolitik“, welcher als ressortübergreifende Querschnittsaufgabe aus Kommunensicht eine stetig wachsende Bedeutung erfährt (Dallmann/Richter 2012: 19). Mit gezielten Maßnahmen sollen die Rahmenbedingungen für wirtschaftliches
Handeln in einer Kommune möglichst positiv beeinflusst werden, um Betriebe an eine Kommune zu binden und eine allgemeine Verbesserung der Lebensbedingungen $\mathrm{zu}$ erreichen (Held/Markert 2001; Markert 2018). Nicht überraschend und in vollständiger Übereinstimmung mit den Ergebnissen dieser Studie, zeigt eine Umfrage zur Bedeutung der Themenfelder kommunaler Wirtschaftsförderung unter Kommunen zwischen 10.000 und 50.000 Einwohner/ -innen, dass die im Rahmen der Modellierung vorgeschlagenen Bereiche der Wirtschaftsförderung (Ausbildungsplätze und Fachkräfte; Stadt-, Standort- und Regionalentwicklung; Förderung von Netzwerken) in den vergangenen Jahren am stärksten an Bedeutung gewonnen haben (Fuchs/Hansen/ Pieckenäcker 2013).

Bebauungspläne, Altstadtsatzungen sowie verkehrsplanerische Konzepte und Maßnahmen der Kommunen können für ortsansässige Betriebe einschränkend oder fördernd wirken, beispielsweise wenn sie notwendige bauliche Veränderungen oder die Passantenfrequenz betreffen. Eine Anpassung kommunaler Festlegungen an die Bedarfe der Betriebe des ortsansässigen, inhabergeführten Lebensmittelhandwerks kann unter dem Begriff der Förderung urbaner Produktion gefasst werden. Hierbei handelt es sich um ein Konzept, welches im Stadtraum ein funktionierendes Miteinander zwischen verarbeitendem Gewerbe und Wohnnutzung und somit eine stärkere Nutzungs- und Funktionsmischung vorsieht (Brandt/Gärtner/Meyer 2017). Das ortsansässige, inhabergeführte Lebensmittelhandwerk als Teil der urbanen Produktion trägt dazu bei, die Nahrungsmittelverarbeitung in den Städten zu erhalten. Für Betriebe in zentraleren Lagen ergibt sich daraus die Notwendigkeit für emissionsärmere und ressourceneffizientere Produktions- und Transportweisen, um Nutzungskonflikte mit Anwohner/-innen zu vermeiden, was grundsätzlich zu einer kommunalen Nachhaltigkeitstransformation beiträgt (Brandt/Gärtner/ Meyer 2017).

Die systemisch abgeleiteten Governance-Interventionen zum Erhalt des ortsansässigen, inhabergeführten Lebensmittelhandwerks und zur Stärkung der lokalen Ökonomie fokussieren auf die Interventionskategorie der Rückkopplungen. Gegenüber Interventionen, die allein auf Parameter wie die Zahl der Betriebe oder Umsatzentwicklung gestützt sind, haben sie den Vorteil, dass systemimmanente Dynamiken mitberücksichtigt werden können. Gleichzeitig bieten sie die Möglichkeit der gezielten Steuerung, die nur auf eine überschaubare Zahl von Betrieben abzielt und das System über Hebelwirkungen aktiviert. Systemsteuerung über Strukturen und vor allem über Paradigmen sind dagegen mit großem Aufwand sowie Unsicherheiten und entsprechend langen Zeiträumen des Systemwandels verbunden, wie das Beispiel der Steuerung kommunaler Ernährungs- 
systeme eindrucksvoll zeigt (Stierand 2008; Baldy 2019; Eckhardt 2020).

Prinzipiell sind die Ergebnisse der vorliegenden Studie auch auf lokale Ökonomien unterschiedlicher Größe skalierbar, da es sich um generelle Systemdynamiken handelt. $\mathrm{Zu}$ berücksichtigen ist jedoch, dass die Modellierung der Dynamiken und die Ableitung von Systemarchetypen und Hebelpunkten für Governance-Interventionen maßgeblich durch die gewählten Systemgrenzen und den Begründungszusammenhang der Analyse geprägt sind (Nabavi/Daniell/Najafi 2017; Eisenack/Villamayor-Tomas/Epstein et al. 2019). Bei der Festlegung der Ziele solcher Steuerungsansätze sowie der Frage nach diesbezüglichen Hebelpunkten muss die jeweilige normative Grundhaltung offengelegt und diskutiert werden. So ist es zum Beispiel aus ökologischer Sicht fraglich, ob die dezentralen Strukturen des ortsansässigen, inhabergeführten Lebensmittelhandwerks überhaupt schützenswert sind, nutzen sie doch in deutlich geringerem Maße als überregional organisierte Großbetriebe sogenannte ökologische Skaleneffekte (vgl. Schlich/Fleissner 2005; Born/ Purcell 2006; Brunori/Galli/Barjolle et al. 2016). Zudem kann eine Entwicklung hin zu filialisierten Strukturen auch Vorteile für die Nahversorgung einer Stadtgesellschaft bieten, wie beispielsweise niedrigere Preise und/oder längere Öffnungszeiten. Andererseits zeigt eine sozioökonomische Perspektive, dass Nachhaltigkeitstransformation und auch diesbezügliche Gestaltbarkeit lokaler Ökonomien auf dem Vorhandensein (lokal)spezifischer Akteursnetzwerke und Branchen wie dem ortsansässigen, inhabergeführten Lebensmittelhandwerk beruht (vgl. Luthe/Wyss/Schuckert 2012; Le Velly/Dufeu 2016; Sipple/Schanz 2019).

Hinzu kommt, dass individuelle Ausprägungen von Systemelementen, vor allem über den Zeitverlauf, deutlich abweichendes Systemverhalten nach sich ziehen können (MacKinnon/Dawley/Pike et al. 2019). So spielen im Kontext von kommunalen Ernährungssystemen beispielsweise unterschiedliche ,Bürgerkulturen“ der betreffenden lokalen Ökonomien (Schanz/Pregernig/Kruse 2020) sowie die verschiedenen Persönlichkeiten in der Leitung der eigentümergeführten Handwerksbetriebe (Rohrlack 2008) eine wichtige Rolle für die Entwicklung der Systeme. Governance-Interventionen zur Steuerung von lokalen Ökonomien anhand der Analyse von Rückkopplungsdynamiken sollten deshalb zunächst eine weitere Überprüfung der spezifisch wahrgenommen Systemvariablen vornehmen.

Aufgrund der komplexen Wirkzusammenhänge von Systemen gilt generell, dass aus Modellierungen abgeleitete Handlungsempfehlungen mit großer Vorsicht genutzt werden sollten. Das Aufzeigen der kausalen Funktionsweise der Kausalschleifendiagramme und ihre flexible Darstellung können zwar Komplexität reduzieren, die Offenlegung und Beschreibung von Rückkopplungen in den Ursache-
Wirkungs-Beziehungen sollten aber nicht mit dem überkommenen Steuerungsoptimismus der 1960er-Jahre verwechselt werden (Sterman 2000). Vielmehr sollte das aufgezeigte Vorgehen als unterstützendes Instrumentarium eines reflexiven Governance-Verständnisses zur Stärkung lokaler Ökonomien Anwendung finden (Mann 2017).

\section{Schlussfolgerung}

Die vorliegende Analyse zeigt, dass neben den generellen Marktmechanismen einer zunehmenden Zentralisierung und Filialisierung vor allem der herrschende Fachkräfteund Nachfolgemangel zum Betrieberückgang im inhabergeführten, ortsansässigen Lebensmittelhandwerk beiträgt. Dieser führt zwar auch zu Nachfragezuwächsen bei den verbliebenen Betrieben, was jedoch in Kombination mit dem herrschende Fachkräftemangel zu einer weiter steigenden Betriebsauslastung führt, welcher nur schwer entgegengewirkt werden kann. Als Reaktion lassen sich betriebsinterne Strategien identifizieren, die zwar wirksam, jedoch aufgrund ungewollter Konsequenzen und verzögerter Wirkungen zum Teil negative Rückkopplungseffekte mit sich bringen. Hier kann kommunale Politik und Verwaltung ansetzen und durch einen kontextsensitiven Einsatz von Instrumenten der Wirtschaftsförderung und Stadtplanung die jeweiligen Strategien der Betriebe unterstützen und deren Erhalt (mit)gewährleisten. Mithilfe einer systemischen Perspektive und der Erstellung und Analyse von Kausalschleifendiagrammen lassen sich hierfür Hebelpunkte für Governance-Interventionen der Stadtentwicklung identifizieren. Über diese kann dem Betrieberückgang in spezifischen Branchen entgegengewirkt und so die Heterogenität lokaler Ökonomien gestärkt werden. Gleichzeitig können die Ziele einer kommunalen Nachhaltigkeitstransformation, wie z. B. eine ökologische/regionale Spezialisierung, vorangebracht werden und inhabergeführte Betriebe als diesbezüglich relevante Ansprechpartner erhalten werden. Eine Ausweitung der Vorgehensweise zur Identifikation von Hebelpunkten kommunaler Governance auf weitere Branchen lokaler Ökonomien erscheint daher möglich und sinnvoll.

Die Ergebnisse der vorliegenden Studie zeigen, dass es entgegen vieler Annahmen (vgl. Pothukuchi/Kaufman 2000; Levkoe 2011; Brinkley 2013) auch auf kommunaler Ebene Möglichkeiten gibt, um eine Nachhaltigkeitstransformation über die Stärkung lokaler Ökonomien zu unterstützen. Bleiben Kommunen hier untätig, werden in vielen Branchen lokaler Ökonomien in naher Zukunft kaum noch dezentrale und individuelle Kleinbetriebe bestehen. Dies hat zumindest in Klein- und Mittelstädten eine Zunahme des Leerstands und Funktionsverlusts der betroffenen Innenstädte und Teilorte zur Folge. Mit einer sichtbar abneh- 
menden Vielfalt lokaler (Nahrungsmittel-)Betriebe schwindet das Potenzial, über lokale Ökonomien eine nachhaltige Stadtentwicklung zu fördern.

\section{Literatur}

Abson, D. J.; Fischer, J.; Leventon, J.; Newig, J.; Schomerus, T.; Vilsmaier, U.; von Wehrden, H.; Abernethy, P.; Ives, C. D; Jager, N. W.; Lang, D.J (2017): Leverage points for sustainability transformation. In: Ambio 46, 1 , 30-39. https://doi.org/10.1007/s13280-016-0800-y

Baldy, J. (2019): Framing a Sustainable Local Food System - How Smaller Cities in Southern Germany Are Facing a New Policy Issue. In: Sustainability 11, 6, 1712. https:// doi.org/10.3390/su11061712

Beckert, J. (2010): How Do Fields Change? The Interrelations of Institutions, Networks, and Cognition in the Dynamics of Markets. In: Organization Studies 31, 5, 605-627. https://doi.org/10.1177/0170840610372184

Beckert, J. (2013): Imagined futures: fictional expectations in the economy. In: Theory and Society 42, 3, 219-240. https://doi.org/10.1007/s11186-013-9191-2

Beile, J.; Drescher-Bonny, I.; Maack, K. (2009): Zukunft des Backgewerbes. Düsseldorf.

Bizer, K.; Haverkamp, K. (2012): Nachhaltigkeit im Handwerk - für eine Strategie in der arbeitsteiligen Weltwirtschaft. In: Bizer, K.; Haverkamp, K. (Hrsg.): Nachhaltigkeit im Handwerk. Duderstadt, 1-12. = Göttinger Handwerkswirtschaftliche Studien 88 .

Born, B.; Purcell, M. (2006): Avoiding the local trap: Scale and food systems in planning research. In: Journal of Planning Education and Research 26, 2, 195-207. https:// doi.org/10.1177/0739456X06291389

Brandt, M.; Gärtner, S. (2019): Lokale Ökonomie - Was, wer, wie auf welchen Ebenen? In: Niermann, O.; Schnur, O.; Drilling. M. (Hrsg.): Ökonomie im Quartier: Von der sozialräumlichen Intervention zur Postwachstumsgesellschaft. Wiesbaden, 29-47. https://doi.org/10.1007/9783-658-23446-1_3

Brandt, M.; Gärtner, S.; Meyer, K. (2017): Urbane Produktion - ein Versuch einer Begriffsdefinition. Gelsenkirchen. $=$ Institut für Arbeit und Technik, Forschung Aktuell 08/2017.

Brinkley, C. (2013): Avenues into Food Planning. A Review of Scholarly Food System Research. In: International Planning Studies 18, 2, 243-266. https://doi.org/10. 1080/13563475.2013.774150

Brunori, G.; Galli, F.; Barjolle, D.; Van Broekhuizen, R.; Colombo, L.; Giampietro, M.; Kirwan, J.; Lang, T.; Mathijs, E.; Maye, D.; De Roest, K.; Rougoor, C.; Schwarz, J.; Schmitt, E.; Smith, J.; Stojanovic, Z.; Tisenkopfs, T.; Touzard, J.-M. (2016): Are local food chains more su- stainable than global food chains? Considerations for assessment. In: Sustainability 8, 5, 449. https://doi.org/10. 3390/su8050449

Chilla, T.; Kühne, O.; Neufeld, M. (2016): Regionalentwicklung. Stuttgart.

Coyle, R. G. (1997): System dynamics modelling: a practical approach. London.

Dallmann, B.; Richter, M. (2012): Handbuch der Wirtschaftsförderung. Praxisleitfaden zur kommunalen und regionalen Standortentwicklung. Freiburg.

Dannenberg, P.; Willkomm, M.; Zehner, K. (2017): Einzelhandel und räumliche Entwicklung - aktuelle Dynamiken in Deutschland. In: Dannenberg, P.; Willkomm, M.; Zehner, K. (Hrsg.): Einzelhandel in Deutschland - Aktuelle Dynamiken im Kontext neuer Rahmenbedingungen. Mannheim, 1-8. = Geographische Handelsforschung 25.

Destatis (Statistisches Bundesamt) (2020): Produzierendes Gewerbe. Beschäftigte und Umsatz im Handwerk - Messzahlen und Veränderungsraten (Vorläufige Ergebnisse). Fachserie 4, Reihe 7.1, 2. Vierteljahr 2019. Wiesbaden.

Deutscher Bundestag (2019): Antwort der Bundesregierung auf die Kleine Anfrage der Abgeordneten Markus Tressel, Claudia Müller, Renate Künast, weiterer Abgeordneter und der Fraktion BÜNDNIS 90/DIE GRÜNEN. Stärkung des regionalen Lebensmittelhandwerks. Berlin. $=$ Drucksache 19/7287.

Doernberg, A.; Horn, P.; Zasada, I.; Piorr, A. (2019): Urban food policies in German city regions: An overview of key players and policy instruments. In: Food Policy 89, 101782. https://doi.org/10.1016/j.foodpol.2019.101782

Dütschke, E.; Köhler, J.; Laws, N.; Hacke, U.; NiedersteHollenberg, J.; Wesche, J. (2019): Kommunen als Motoren einer Nachhaltigkeitstransformation - Erfahrungen aus den Feldern Energie, Wasser und Wohnen. In: Leal Filho, W. (Hrsg.): Aktuelle Ansätze zur Umsetzung der UN-Nachhaltigkeitsziele. Heidelberg, 79-98. https://doi. org/10.1007/978-3-662-58717-1_5

Eckhardt, T. (2020): Welche Ressourcen sind innerhalb einer Verwaltung notwendig, um eine Nachhaltigkeitstransformation im Ernährungsbereich anzustoßen? Politischer Wille und Zeit. In: Schanz, H.; Pregernig, M.; Baldy, J.; Sipple, D.; Kruse, S. (Hrsg.): Kommunen gestalten Ernährung - Neue Handlungsfelder nachhaltiger Stadtentwicklung. Berlin. = Deutscher Städte- und Gemeindebund Dokumentationen 153. https://doi.org/ 10.6094/UNIFR/154838

Eisenack, K.; Villamayor-Tomas, S.; Epstein, G.; Kimmich, C.; Magliocca, N.; Manuel-Navarrete, D.; Oberlack, C.; Roggero, M.; Sietz, D. (2019): Design and quality criteria for archetype analysis. In: Ecology and Society 24, 3, 6. https://doi.org/10.5751/ES-10855-240306 
Fesenfeld, L. P. (2016): Governing Urban Food Systems in the Long Run: Comparing Best Practices in Sustainable Food Procurement Regulations. In: GAIA - Ecological Perspectives for Science and Society 25, 4, 260-270. https://doi.org/10.14512/gaia.25.4.8

Flick, U. (2012): Qualitative Sozialforschung. Eine Einführung. Reinbek.

Forrester, J. W. (1969): The City. Urban Dynamics. Cambridge.

Franz, M. (2020): Gaststätten als Teil der lokalen Ökonomie. In: Henn, S.; Behling, M.; Schäfer, S. (Hrsg.): Lokale Ökonomie - Konzepte, Quartierskontexte und Interventionen. Berlin, 191-209. https://doi.org/10.1007/9783-662-57780-6_14

Freeman, R.; Yearworth, M.; Preist, C. (2016): Revisiting Jevons' Paradox with System Dynamics: Systemic Causes and Potential Cures. In: Journal of Industrial Ecology 20, 2, 341-353. https://doi.org/10.1111/jiec.12285

Fuchs, T.; Hansen, C.; Pieckenäcker, B. A. (2013): Aufgaben, Organisation und Schwerpunkte der kommunalen Wirtschaftsförderung. Berlin. = Deutscher Städte- und Gemeindebund Dokumentation 118.

Fülles, M.; Roehl, R.; Strassner, C.; Hermann, A.; Teufel, J. (2017): Mehr Bio in Kommunen: Ein Praxisleitfaden des Netzwerks deutscher Biostädte. Nürnberg.

Gläser, J.; Laudel, G. (2010): Experteninterviews und qualitative Inhaltsanalyse. Wiesbaden.

Haverkamp, K. (2011): Nachfragepotenziale und Absatzchancen des Handwerks im demografischen Wandel. Duderstadt. = Göttinger Handwerkswirtschaftliche Arbeitshefte 69.

Held, H.; Markert, P. (2001): Wirtschaftsförderung in Deutschland - Status quo und Ausblick. In: Institut für Marketing und Kommunalentwicklung (Hrsg.): Wirtschaftsförderung im 21. Jahrhundert. Konzepte und Lösungen. Aalen, 5-24. = Schriften zur Wirtschaftsförderung 1.

Helmle, S. (2005): Lokale Ökonomie. In: Beetz, S.; Brauer, K.; Neu, C. (Hrsg.): Handwörterbuch zur ländlichen Gesellschaft in Deutschland. Wiesbaden, 153-159. https:// doi.org/10.1007/978-3-322-80909-4_19

Henn, S.; Behling, M. (2020): Lokale Ökonomie - Begriff, Merkmale und konzeptionelle Abgrenzung. In: Henn, S.; Behling, M.; Schäfer, S. (Hrsg.): Lokale Ökonomie Konzepte, Quartierskontexte und Interventionen. Berlin, 3-24. https://doi.org/10.1007/978-3-662-57780-6_1

Hilpert, M.; Völkening, N.; Beck, C. (2018): Innenstädte im ländlichen Raum. Wirksame Maßnahmen zur Revitalisierung zentraler Einzelhandelslagen im ruralen Umfeld. In: Standort - Zeitschrift für Angewandte Geographie 42, 2, 111-116. https://doi.org/10.1007/s00548-018-0532-2 Hirschnitz-Garbers, M.; Distelkamp, M.; Koca, D.; Meyer,
M.; Sverdrup, H. (2018): Potentiale und Kernergebnisse der Simulationen von Ressourcenschonung(spolitik). Endbericht des Projekts ,Modelle, Potentiale und Langfristszenarien für Ressourceneffizienz“. Dessau-Roßlau. $=$ UBA-Texte 48/2018.

Holtz, G.; Alkemade, F.; de Haan, F.; Köhler, J.; Trutnevyte, E.; Luthe, T.; Halbe, J.; Papachristos, G.; Chappin, E.; Kwakkel, J.; Ruutu, S. (2015): Prospects of modelling societal transitions: Position paper of an emerging community. In: Environmental Innovation and Societal Transitions 17, 41-58. https://doi.org/10.1016/j.eist.2015.05. 006

Jakubowski, P.; Koch, A. (2009): Stärkung der lokalen Ökonomie - eine stadtentwicklungspolitische Aufgabe. In: Informationen zur Raumentwicklung 3/4, 241-253.

Kim, D. H. (1992): Toolbox: Guidelines for Drawing Causal Loop Diagrams. In: The Systems Thinker 3, 1, 5-6.

Kögl, H.; Tietze, J. (2010): Regionale Erzeugung, Verarbeitung und Vermarktung von Lebensmitteln. Abschlussbericht. Studie im Auftrag des Bundesministeriums für Landwirtschaft, Ernährung und Verbraucherschutz. Rostock.

Kulke, E. (2017): Wirtschaftsgeographie. Stuttgart.

Läpple, D.; Walter, G. (2003): Lokale Ökonomie. Arbeiten und Produzieren im Stadtteil. In: StadtBauwelt 157, 12, 24-33.

Le Velly, R.; Dufeu, I. (2016): Alternative food networks as "market agencements": Exploring their multiple hybridities. In: Journal of Rural Studies 43, 173-182. https:// doi.org/10.1016/j.jrurstud.2015.11.015

Leal Filho, W. (2019): Die Nachhaltigkeitsziele der UN: eine Chance zur Vermittlung eines besseren Verständnisses von Nachhaltigkeitsherausforderungen. In: Leal Filho, W. (Hrsg.): Aktuelle Ansätze zur Umsetzung der UN-Nachhaltigkeitsziele. Heidelberg, 1-20. https://doi. org/10.1007/978-3-662-58717-1_1

Levkoe, C. Z. (2011): Towards a transformative food politics. In: Local Environment 16, 7, 687-705. https://doi. org/10.1080/13549839.2011.592182

Luederitz, C.; Abson, D. J.; Audet, R.; Lang, D. J. (2017): Many pathways toward sustainability: Not conflict but co-learning between transition narratives. In: Sustainability Science 12, 3, 393-407. https://doi.org/10.1007/ s11625-016-0414-0

Luthe, T.; Wyss, R.; Schuckert, M. (2012): Network governance and regional resilience to climate change: empirical evidence from mountain tourism communities in the Swiss Gotthard region. In: Regional Environmental Change 12, 4, 839-854. https://doi.org/10.1007/s10113012-0294-5

MacKinnon, D.; Dawley, S.; Pike, A.; Cumbers, A. (2019): Rethinking Path Creation: A Geographical Political Eco- 
nomy Approach. In: Economic Geography 95, 2, 113135. https://doi.org/10.1080/00130095.2018.1498294

Mann, C. (2017): Governance von Gesellschaftstransformation: Konzeptionelle Überlegungen und eine Momentaufnahme politischer Initiativen und Maßnahmen in Deutschland. In: Rückert-John, J.; Schäfer, M. (Hrsg.): Governance für eine Gesellschaftstransformation: Herausforderungen des Wandels in Richtung nachhaltige Entwicklung. Wiesbaden, 13-43. https://doi.org/10. 1007/978-3-658-16560-4_1

Markert, P. (2018): Wirtschaftsförderung und Standortmarketing. In: Meffert, H.; Spinnen, B.; Block, J. (Hrsg.): Praxishandbuch City- und Stadtmarketing. Wiesbaden, 205-223. https://doi.org/10.1007/978-3-658-19642-4_ 14

Mayring, P. (2010): Qualitative Inhaltsanalyse. In: Mey, G.; Mruck, K. (Hrsg.): Handbuch Qualitative Forschung in der Psychologie. Wiesbaden, 601-613. https://doi.org/10. 1007/978-3-531-92052-8_42

Meadows, D. (1999): Leverage points: places to intervene in a system. Hartland. http://donellameadows. org/archives/leverage-points-places-to-intervene-in-asystem/ (09.12.2020).

Meadows, D. (2019): Die Grenzen des Denkens: Wie wir sie mit System erkennen und überwinden können. München.

Meier Kruker, V.; Rauh, J. (2005): Arbeitsmethoden der Humangeographie. Darmstadt.

Mingers, J. (2014): Systems thinking, critical realism and philosophy: A confluence of ideas. London. https://doi. org/10.4324/9781315774503

Moll, C. (2019): Nachhaltige Dienstleistungsinnovationen in der Logistik: Ein Ansatz zur Entwicklung von Entscheidungsmodellen. Wiesbaden. https://doi.org/10. 1007/978-3-658-28038-3

Mossig, I.; Dorenkamp, A. (2010): Shopping-Malls und Business Improvement Districts als Instrumente zur Belebung innerstädtischer Geschäftszentren? Das Beispiel der Stadt Gießen. Bremen. = Beiträge zur Wirtschaftsgeographie und Regionalentwicklung 2.

Nabavi, E.; Daniell, K. A.; Najafi, H. (2017): Boundary matters: the potential of system dynamics to support sustainability? In: Journal of Cleaner Production 140, 1, 312-323. https://doi.org/10.1016/j.jclepro.2016.03.032

Oberlack, C.; Sietz, D.; Bürgi Bonanomi, E.; de Bremond, A.; Dell'Angelo, J.; Eisenack, K.; Ellis, C. E.; Epstein, G.; Giger, M.; Heinimann, A.; Kimmich, C.; Kok, M. T. J.; Manuel-Navarrete, D.; Messerli, P.; Meyfroidt, P.; Václavik, T; Villamayor-Tomas, S. (2019): Archetype analysis in sustainability research. Meanings, motivations, and evidence-based policy making. In: Ecology and
Society 24, 2, 26. https://doi.org/10.5751/ES-10747240226

Pothukuchi, K.; Kaufman, J. L. (2000): The Food System. A Stranger to the Planning Field. In: Journal of the American Planning Association 66, 2, 113-124. https://doi. org/10.1080/01944360008976093

Proeger, T.; Thonipara, A.; Bizer, K. (2020): Mechanismen, Erfolgsfaktoren und Hemmnisse der Digitalisierung im Handwerk. Göttingen. = Göttinger Beiträge zur Handwerksforschung 35. https://doi.org/10.3249/2364-3897gbh-35

Rädiker, S.; Kuckartz, U. (2019): Analyse qualitativer Daten mit MAXQDA. Wiesbaden. https://doi.org/10.1007/9783-658-22095-2

Reichertz, J. (2013): Die Abduktion in der qualitativen Sozialforschung: Über die Entdeckung des Neuen. Wiesbaden. https://doi.org/10.1007/978-3-531-93163-0

Reiter, A. (2018): Perspektiven für das Stadtmarketing von morgen. In: Meffert, H.; Spinnen, B.; Block, J. (Hrsg.): Praxishandbuch City- und Stadtmarketing. Wiesbaden, 3-10. https://doi.org/10.1007/978-3-658-19642-4_1

Richardson, G. P. (2011): Reflections on the foundations of system dynamics. In: System Dynamics Review 27, 3, 219-243. https://doi.org/10.1002/sdr.462

Rohn, H. (2012): Nachhaltige Ernährungswirtschaft und Ernährung: Herausforderungen und Chancen für das Lebensmittelhandwerk. In: Bizer, K.; Haverkamp, K. (Hrsg.): Nachhaltigkeit im Handwerk. Duderstadt, 125144. = Göttinger Handwerkswirtschaftliche Studien 88 .

Rohrlack, K. (2008): Analyse des betrieblichen Verhaltens von Handwerksbetrieben im Hinblick auf demographische Entwicklungen. München. = Schriftenreihe Empirische Personal- und Organisationsforschung 37.

Runst, P.; Haverkamp, K. (2018): Handwerk im ländlichen Raum. Göttingen. = Göttinger Beiträge zur Handwerksforschung 22. https://doi.org/10.3249/2364-3897-gbh22

Santarius, T. (2014): Der Rebound-Effekt: ein blinder Fleck der sozial-ökologischen Gesellschaftstransformation. In: GAIA - Ecological Perspectives for Science and Society 23, 2, 109-117. https://doi.org/10.14512/gaia.23.2.8

Schanz, H.; Pregernig, M.; Baldy, J.; Sipple, D.; Kruse, S. (Hrsg.) (2020): Kommunen gestalten Ernährung - Neue Handlungsfelder nachhaltiger Stadtentwicklung. Berlin. $=$ Deutscher Städte- und Gemeindebund Dokumentationen 153. https://doi.org/10.6094/UNIFR/154838

Schanz, H.; Pregernig, M.; Kruse, S. (2020): Wie läuft die Umsetzung konkret? Einschätzungen aus Sicht der Wissenschaft. In: Schanz, H.; Pregernig, M.; Baldy, J.; Sipple, D.; Kruse, S. (Hrsg.): Kommunen gestalten Ernährung - Neue Handlungsfelder nachhaltiger Stadtentwicklung. Berlin, 30. = Deutscher Städte- und Gemeindebund 
Dokumentationen 153. https://doi.org/10.6094/UNIFR/ 154838

Schlich, E.; Fleissner, U. (2005): The Ecology of Scale. Assessment of Regional Energy Turnover and Comparison with Global Food. In: The International Journal of Life Cycle Assessment 10, 3, 219-223. https://doi.org/10. 1065/lca2004.09.180.9

Senge, P. M. (2011): Die fünfte Disziplin - Kunst und Praxis der lernenden Organisation. Stuttgart.

Setianto, N. A.; Cameron, D.; Gaughan, J. B. (2014): Identifying archetypes of an enhanced system dynamics causal loop diagram in pursuit of strategies to improve smallholder beef farming in Java, Indonesia. In: Systems Research and Behavioral Science 31, 5, 642-654. https:// doi.org/10.1002/sres.2312

Sipple, D.; Schanz, H. (2019): Nachhaltige Stadtentwicklung über kommunale Ernährungssysteme: Marktakteursnetzwerke als Ansatzpunkte zur Gestaltung und Steuerung. In: Zeitschrift für Wirtschaftsgeographie 63, 1, 1-22. https://doi.org/10.1515/zfw-2018-0024

Sloane, A.; O'Reilly, S. (2013): The emergence of supply network ecosystems: a social network analysis perspective. In: Production Planning and Control 24, 7, 621-639. https://doi.org/10.1080/09537287.2012.659874

Smith, A.; Raven, R. (2012): What is protective space? Reconsidering niches in transitions to sustainability. In: Research Policy 41, 6, 1025-1036. https://doi.org/10.1016/ j.respol.2011.12.012

Spars, G.; Busch, R. (2020): Strukturen der lokalen Ökonomie im deutschen Städtevergleich. In: Henn, S.; Behling, M.; Schäfer, S. (Hrsg.): Lokale Ökonomie - Konzepte, Quartierskontexte und Interventionen. Berlin, 2536. https://doi.org/10.1007/978-3-662-57780-6_2

Sperle, T. (2012): Was kommt nach dem Handel? Umnutzung von Einzelhandelsflächen und deren Beitrag zur Stadtentwicklung. Dissertation an der Universität Stuttgart. https://doi.org/10.18419/opus-93

Statista (2019a): Bäckerhandwerk in Deutschland. Dossier. Hamburg.

Statista (2019b): Fleischerhandwerk in Deutschland. Dossier. Hamburg.

Statistisches Landesamt Baden-Württemberg (2017): Agrar- strukturerhebung 2016in Baden-Württemberg. Stuttgart. $=$ Statistische Daten 05/2017.

Sterman, J. (2000): Business Dynamics: Systems Thinking and Modeling for a Complex World. Boston.

Stierand, P. (2008): Stadt und Lebensmittel: die Bedeutung des städtischen Ernährungssystems für die Stadtentwicklung. Dissertation an der Technischen Universität Dortmund.

Storbacka, K.; Nenonen, S. (2011): Scripting markets: From value propositions to market propositions. In: Industrial Marketing Management 40, 2, 255-266. https://doi.org/ 10.1016/j.indmarman.2010.06.038

Sverdrup H.; Haraldsson, H.; Olafsdottir, A.H.; Belyazid, S.; Svensson, M. (2018): System Thinking, System Analysis and System Dynamics: Find out how the world works and then simulate what would happen. Reykjavik.

Ulm, H. (2007): Tote Hose Innenstadt? Das Problem der verödenden Ortskerne in Städten und Dörfern des ländlichen Raums. In: Mitteilungen der Fränkischen Geographischen Gesellschaft 53/54, 153-168.

van Raak, R. (2015): Transition policies. Connecting system dynamics, governance and instruments in an application to Dutch healthcare. Rotterdam.

Vereinte Nationen (2015): Resolution der Generalversammlung, verabschiedet am 25. September 2015: Transformation unserer Welt: die Agenda 2030für nachhaltige Entwicklung. New York.

Weck, S. (2012): Lokale Ökonomie: Ein lohnendes Handlungsfeld. In: Ministerium für Bauen, Wohnen, Stadtentwicklung und Verkehr des Landes Nordrhein-Westfalen (Hrsg.): Lokale Ökonomie in Nordrhein-Westfalen: Wirt-

schaft - Beschäftigung - Qualifizierung - Image. Düsseldorf, 8-13.

Wolstenholme, E. F. (2003): Towards the definition and use of a core set of archetypal structures in system dynamics. In: System Dynamics Review 19, 1, 7-26. https://doi.org/ 10.1002/sdr.259

zur Nedden, M.; Bunzel, A.; Pätzold, R.; Strauss, W.-C.; Spars, G.; Busch, R.; Heinze, M.; Müller, A. (2015): Nutzungsmischung und soziale Vielfalt im Stadtquartier - Bestandsaufnahme, Beispiele, Steuerungsbedarf. Endbericht. Berlin. 